\title{
Non-invasive assessment of arterial distension waveforms using gradient-based Hough transform and power Doppler ultrasound imaging
}

\author{
$\begin{array}{llll}\text { S.-M. Wu } & \text { Y., W. Shau } & \text { F.-C. Chong } & \text { F.-J. Hsieh } \\ & & \end{array}$ \\ ${ }^{1}$ College of Electrical Engineering, Biomedical Engineering, National Taiwan University, Taipei, Taiwan \\ ${ }^{2}$ Institute of Applied Mechanics, Biophysics Laboratory, National Taiwan University, Taipei, Taiwan \\ ${ }^{3} \mathrm{Jin}-$ Wen Institute of Technology, Hsin-Tien, Taipei Hsien, Taiwan \\ ${ }^{4}$ Department of Diagnostic Ultrasound, National Taiwan University Hospital, Taipei, Taiwan
}

\begin{abstract}
The peripheral arterial vessel often appears as an elliptic shape under the constraints of the surrounding tissues. In this study, the gradient-based Hough transform was used to detect the central location of the ellipse and the lumen area of the arterial vessel non-invasively using power Doppler imaging. Sequential ultrasound images were used to construct arterial distension waveforms in both the major- and minor-axis directions for a few cardiac cycles. The common carotid arteries (CCAs) for nine healthy male volunteers (mean age 24 years), in the sitting position, were investigated in vivo. The CCAs $(n=9)$ had a mean diameter of $5.83 \mathrm{~mm}$, and the pulsatile diameter distension was $13.7 \pm 1.9 \%$. The brachial artery and dorsalis pedis artery for five healthy male volunteers (mean age 26 years), in the supine position, had mean diameters of $4.03 \mathrm{~mm}$ and $2.83 \mathrm{~mm}$ and distensions of $16.7 \pm 4.6 \%$ and $15.5 \pm 5.4 \%$, respectively. The movement of the arterial centre location during the cardiac cycle reflected the asymmetry of the reaction forces produced by the surrounding soft tissues. The present method can obtain the response of vessel distension to pulse pressure, as well as the constrained conditions of the arteries.
\end{abstract}

Keywords-Hough transform, Power Doppler imaging, Arterial distension waveforms

Med. Biol. Eng. Comput., 2001, 39, 627-632

\section{Introduction}

IN VIVO measurements of the response of the arterial lumen area to pulse pressure are important in the evaluation of cardiovascular diseases, such as atherosclerosis, hypertension and diabetes. In the literature, non-invasive ultrasound measurements of the arterial distension waveform have been taken using the longitudinal B-mode, M-mode images and echotracking of digitised radio-frequency data (STADLER et al., 1996; 1997; BRANDS et al., 1996; NAGAI et al., 1999). By combining simultaneous measurements of the arterial distension waveforms and the pulse pressure, the arterial elastic modulus and the energy dissipation ratio can be quantified intact (SHAU et al., 1999).

The arterial diameters obtained using the longitudinal B-mode, transverse M-mode views and echo-tracking of vascular walls are accurate only if the lumens are circular in geometry. Unfortunately, according to our clinical observation, the cross-sections of human peripheral arteries often appear elliptical in shape, owing to the variations in stiffness of the

Correspondence should be addressed to Dr Yio-Wha Shau; email: ywshau@spring.iam.ntu.edu.tw

Paper received 5 April 2001 and in final form 27 September 2001

MBEC online number: 20013626

(C) IFMBE: 2001 surrounding tissues (Fig. 1). Moreover, the arterial vessels, in some circumstances, are oblique to the M-line selected. Therefore extracting the correct arterial diameter distension tends to be more difficult.

For an artery of elliptic geometry, the vascular motion must be measured both in the major- and minor-axis directions. It seems that, under the action of pulse pressure, the arterial diameter distensions are different in the minor-axis and major-axis directions (SHAU et al., 1999). Therefore a strategy for arterial lumen-determination that deals with the transverse B-mode view of the arterial vessel is needed.

The Hough transform (HT) has been used successfully to characterise straight-line segments (HoUGH, 1962) and can be extended to generalised features, such as circles and ellipses, for industrial applications (ILLINGWORTH and KITTLER, 1988; YIP et al., 1992; NAIR et al., 1996). It converts the complex global detection problem in image space into simple local-peak detection in parametric space.

Quantifying an ellipse in an image involves five parameters, namely, the centre position $\left(x_{c}, y_{c}\right)$, the orientation $\theta$, and the major and minor axes $(a, b)$. However, the direct extension of the Hough transform to huge five-parameter space is generally slow and difficult.

Many modified Hough transforms have been presented to decompose the geometric parameter space by voting for the centre first and then the axes and the orientation (NAIR et al., 1996; AguAdo et al., 1996; LEI and WONG, 1999). The heavy 


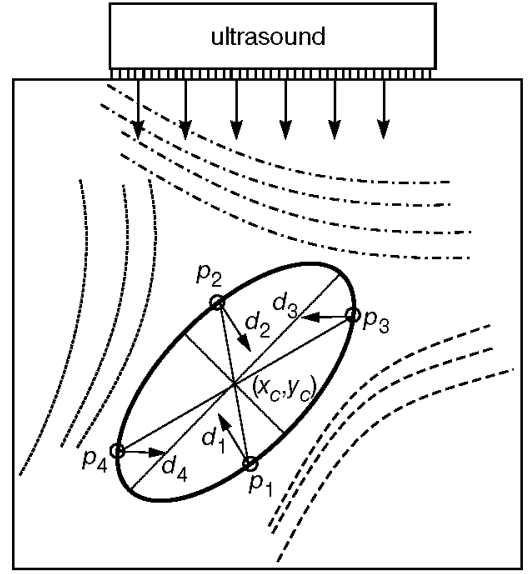

Fig. 1 Schematic diagram of arterial vessel that appears as ellipse owing to constraints of surrounding tissues. Geometric symmetry of ellipse is illustrated by gradient vectors $d_{1} / / d_{2}$, $d_{3} / / d_{4}$ at edge pairs $p_{1}-p_{2}, p_{3}-p_{4}$, respectively, and mid-points for edge pairs cluster at centre $\left(x_{0}, y_{c}\right)$

noise would enhance the disturbance in the voting space and cause forgery peaks; however, the algorithm for finding the large ellipse was less affected (ILLINGWORTH and KITTLER, 1988; AGUADO et al., 1996; LEI and WONG, 1999).

By using the arterial lumen contour of the ultrasound image and fitting it with an ellipse, the vessel shape and orientation and the lumen area can be revealed. Colour Duplex ultrasound imaging would further simplify the process of distinguishing arterial vessels from the surrounding tissues (WOLF and FOBBE, 1994).

In clinical ultrasound, power Doppler imaging (PDI) generates intravascular colour signals that are proportional to the fraction of moving blood within the sample volume (STEINKE et al., 1996; GRIEWING et al., 1996). From the PDI, we can obtain the cross-sectional area of blood flow for large arteries with low levels of inaccuracy (STEINKE et al., 1997; HosKINS et al., 1998; DEVERSON and EVANS, 2000).

This study applied the gradient-based Hough transform to detect the arterial vessels that appeared in a power Doppler image. A set of masks consisting of 32 gradient orientations were used to find the centre of the arteries. The size and the orientation of the artery can be found based on the raw edges and the centre co-ordinates. As the arterial vessel deforms according to the actions of pulse pressure and the surrounding constraints, by applying the algorithm to the sequential PDI of the artery, we can determine the movement of the arterial centre co-ordinates and the arterial distension waveforms both in the major and minor axes during the cardiac cycle.

\section{Methods}

\subsection{Power Doppler imaging and arterial raw edge detection}

The PDI was obtained using a high-resolution ultrasound system* $^{*}$ that was equipped with a linear array transducer ${ }^{\dagger}$. The transducer was positioned to provide a transverse view of the arterial vessel. The ultrasound scanning lines were focussed on the superficial arterial vessels (brachial artery (BA), dorsalis pedis artery (DPA) and common carotid artery (CCA)), typically located at a depth of 6-25 $\mathrm{mm}$ from the skin surface.

The colour power angio (CPA) level of the ultrasound was set at $81-85 \%$, the line density was set at ' $\mathrm{B}$ ', and the persistence was set at low to give better temporal resolution of the wave-

* HDI 5000, ATL, Bothell, Washington, USA

${ }^{\dagger} \mathrm{CL} 12-538 \mathrm{~mm}$ ATL form. The pulse repetition frequency (PRF) was set to 500$700 \mathrm{~Hz}$, and the wall filter was set to middle; this resulted in a PDI frame rate of about $16-19 \mathrm{~Hz}$. Typically, the number of ultrasound frames taken sequentially corresponded to 2-3 cardiac cycles, at least.

A total of nine healthy male volunteers, aged $24 \pm 6$ years, were recruited for this study. Their average height was $170 \mathrm{~cm}$, and their average weight was $66 \mathrm{~kg}$. Each gave informed consent to participate in the experiments after receiving a full explanation of the test procedures. Volunteers were asked to relax their muscles, in a sitting position, for $10 \mathrm{~min}$ and to refrain from breathing during CCA measurements. The BA and DPA were measured in five subjects, with a mean age of 26 years, in a supine position. Because holding the ultrasound transducer in the hand caused irregular movement artifacts in the PDI, we used a mechanical arm to keep the transducer in place.

A digitised PDI pixel consists of three components: red, green and blue colours (RGB); the PDI pixels where Doppler movements are detected have a higher red $(R)$ value, and the pixels with no noticeable blood movement have equal RGB components $(R=G=B)$. A threshold $(T H=R-B)$ of 20 was selected to outline the vessel boundary and provide the raw edge points for pattern recognition.

The size of the blood vessel can be scaled to its physical length based on the scanning depth ruler provided on the power Doppler image. In this study, the arterial vessels were visualised using PDI with high-density zooming, and the image scale for the CCA corresponded to $0.04762 \mathrm{~mm} \mathrm{pixel}^{-1}$.

\subsection{Gradient-based Hough transform}

The gradient-based Hough transform for arterial-vessel detection is shown schematically in Fig. 2. The process consists of three steps:

Step 1: Edge-pair detection: First, we calculated the edge gradient for each raw edge point $y(i, j)$ using 32 masks $g_{0^{\circ}}(i, j), g_{11.25^{\circ}}(i, j), \ldots, g_{360^{\circ}}(i, j)$, of size $7 \times 7$, that are oriented from $0^{\circ}$ to $360^{\circ}$ at an increment of $11.25^{\circ}$ (JAIN, 1989)

$$
\begin{aligned}
g_{0^{\circ}}(i, j)= & \frac{1}{3} \cdot\left[\frac{y(i, j+1)-y(i, j-1)}{2}\right. \\
& \left.+\frac{y(i, j+2)-y(i, j-2)}{4}+\frac{y(i, j+3)-y(i, j-3)}{6}\right]
\end{aligned}
$$

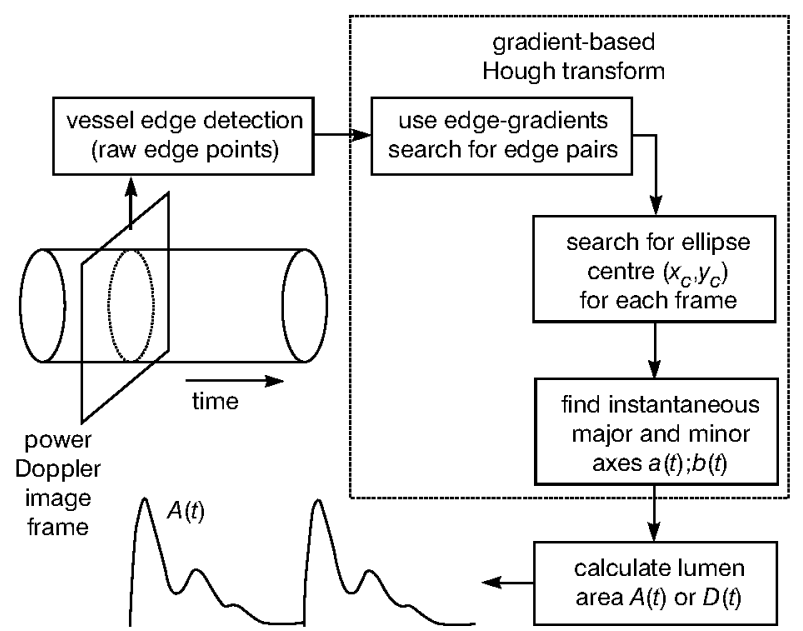

Fig. 2 Schematic diagram of present methodology for arterial distension waveforms 


$$
\begin{aligned}
g_{0^{\circ}}(i, j)= & \frac{1}{36} \cdot[6 y(i, j+1)+3 y(i, j+2)+2 y(i, j+3) \\
& -6 y(i, j-1)-3 y(i, j-2)-2 y(i, j-3)] \\
g_{0^{\circ}}(i, j)= & \sum_{m=-3}^{3} w_{y(i, j+m)} \cdot y(i, j+m)
\end{aligned}
$$

where $w_{y}$ denotes the coefficients of masks. The edge-gradient direction was determined from the mask that resulted in the maximum magnitude. Two edge points that have gradient vectors opposite to each other were grouped to be an edge pair.

Step 2: Centre accumulator: As the edge pairs for an ellipse are symmetrical with respect to the centre, the midpoint of an edge pair would be a potential candidate for the elliptic centre (NAIR and SAUNDERS, 1996). We constructed an accumulator array that corresponded to the mapping of the midpoints of all edge pairs. Any local peak with more counts than the voting threshold indicated a possible instance of the search for an ellipse shape. As the arterial vessel appeared to be the largest ellipse in the image, the pixel at which the maximum histogram of the midpoint for all edge pairs accumulated was voted as the centre $\left(x_{c}, y_{c}\right)$ of the artery (Fig. 1). Details of the algorithm for ellipse detection based on symmetry can be found in LEI and WONG (1999).

Step 3: Major-and minor-axis finder: We then calculated the radial distances $R_{e}$ of each raw edge point from the centre $\left(x_{c}, y_{c}\right)$

$$
R_{e}=\sqrt{\left(x_{e}-x_{c}\right)^{2}+\left(y_{e}-y_{c}\right)^{2}}
$$

By searching the two peaks of the M-shape distance $\left(R_{e}\right)$ histogram (Fig. 3d), the most probable major axis $a$ and minor axis $b$ can be found. The area of the ellipse is simply given by $A=\pi a b$. Furthermore, the equivalent diameter of the arterial vessel can be estimated as $D=2 \sqrt{a b}$.

By detecting the arterial geometry for the sequential PDI frames recorded over a few cardiac cycles, we can construct the arterial area waveform $A(n)$, where $n$ is the frame number. As the image frame rate is known a priori, the frame step can be scaled to the time increment. Therefore the changes of major axis $a$ and minor axis $b$ with respect to time can be estimated. A computer program was written in $\mathrm{C}++$ to extract the arterial distension waveform and the geometric parameters automatically.

\section{Results}

Fig. 3 shows the arterial image-detection process for a CCA. For a given PDI image, the blood vessel can appear as an ellipse (Fig. $3 a$ ). As the power Doppler reveals the locations with blood movement, the edge points of the CCA can be detected by the zero-crossings for a given threshold in the red colour level (Fig. $3 b$ ). Fig. $3 c$ shows the clustering of the edge pairs, and the ellipse centre location $(103,152)$ of the vessel is voted to be the maximum in the $3 \mathrm{D}$ histogram. The distances from the edge points to the centre (radius distribution) were clustered as two values, 62, 60, that denoted the possible major axis and minor axis, respectively (Fig. $3 d$ ). Thus the major-axis and minor-axis physical lengths at this instant are $a=2.95 \mathrm{~mm}$ and $b=2.86 \mathrm{~mm}$, respectively. Fig. $3 e$ shows the ellipse detected using the gradient-based Hough transform, with the original PDI for comparison.

Fig. $4 a$ shows the results of arterial distension waveforms: the CCA vessel deforms in both the major-axis and minor-axis directions. The changes in the $\mathrm{CCA}$ lumen area during the cardiac cycle, calculated based on the ellipse geometry, is shown in Fig. $4 b$. Both the major axis (a) and the minor axis (b) distended simultaneously with arterial pressure; however, the changes in magnitude were slightly different. The arterial lumen area waveform resembles the pulse pressure measured on the CCA vessel locally.

Fig. $5 a$ depicts the movement of the BA centre location recorded during two cardiac cycles. The variations in the major axis $(a)$ and the minor axis $(b)$ with the cardiac cycle are plotted in Fig. 5b, and the arterial lumen area waveform is shown in Fig. 6. Table 1 summarises the results of CCA $(n=9)$, BA and DPA $(n=5)$ diameters and arterial strains for healthy subjects.

\section{Discussion}

The Hough transform has been recognised as a robust method for the detection of circles and ellipses in industrial digital images. It is particularly useful for handling partially occluded and noise-corrupted objects (NAIR and SAUNDERS, 1996; LEI and WONG, 1999). Ultrasound imaging provides an excellent method of visualising blood vessels non-intrusively; however, the posterior wall boundary of the arterial vessel cannot always be well defined. To quantify the arterial diameter distension with reasonable accuracy requires tremendous efforts (STADLER et al., 1996; 1997)

In this study, the geometry of an arterial vessel visualised using PDI was traced and curve-fitted sequentially through a few cardiac cycles. The raw edge points of the arterial lumen extracted from the PDI reduced the numerical calculation size substantially.

As an ellipse exhibits symmetrical edge gradients that lead to its centre co-ordinates, we designed 32 masks to find the edge pairs with better resolution. A blood vessel that demonstrates an ellipse pattern can be quantified automatically and efficiently using image-processing software. The major axis and minor axis were measured so that the arterial lumen area could be calculated, and the difference between the major and minor axes revealed how the surrounding muscles compressed the arterial vessel. The noise in the ultrasound image could disturb the search for true edge pairs and smear the peaks in the voting space. The major- and minor-axis lengths could be altered by up to $2-3 \%$. However, the centre location of the ellipse was less affected. Also, by using higher-resolution gradient masks $(n=32)$, the noise tolerance of the ellipse detection is increased.

The CCA diameter and the arterial strain measured in this study agree reasonably well with our previous work (SHAU et al., 1999). We can further measure the arterial mechanical properties using the arterial distension waveform and the pulse pressure recorded simultaneously. However, to obtain a better representation of the arterial vessel geometry, the ultrasound linear array transducer is recommended.

Traditional arterial wall-tracking techniques using ultrasound are effective for circular vessels, and the arterial vessels are assumed to deform isotropically. However, we find that human peripheral arteries are often surrounded by asymmetric confinements (fatty tissues, muscles and bone), and the arterial vessels can appear in ellipse and be oriented obliquely to the ultrasound scanning line. Further, arterial wall lesion or stiffening can develop locally (atherosclerosis). Therefore, under the same action of pulse pressure, the distension of the arterial wall varies circumferentially with local vessel stiffness and constrained conditions.

In this study, arterial diameter distension was found to have a preferred direction that appeared to be larger in the minor-axis direction than that in the major-axis direction. For a nearly circular arterial vessel, the difference in distension magnitudes is reduced for major and minor axes.

The present method can be used to verify local vessel wall stiffening or lesion by observation of the asymmetry of the vessel wall distension vector. The present approach can also reveal the central movement of the arterial vessel that results 

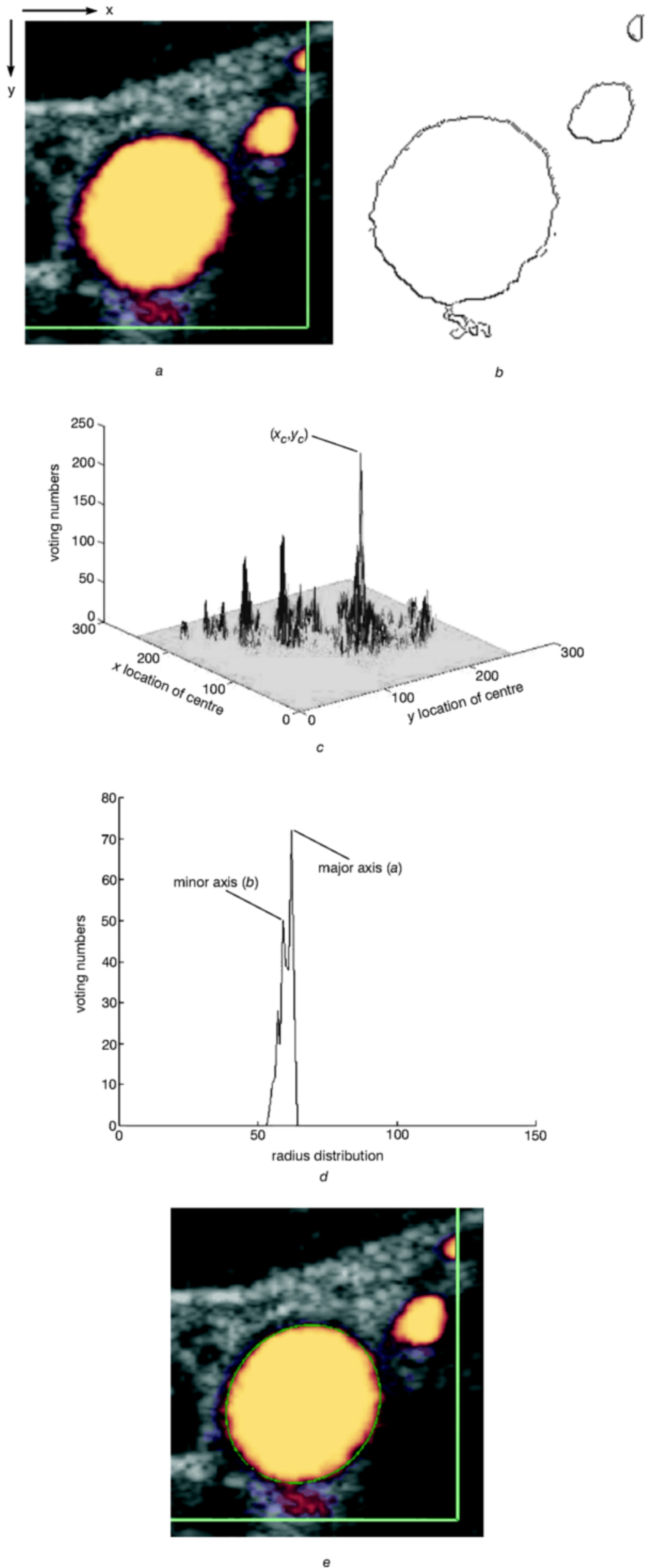

Fig. 3 Demonstration of gradient-based Hough transform for detection of CCA location and size: (a) power Doppler image of CCA vessel $\left(256 \times 256\right.$ pixels); (b) raw edge points detected by the colour threshold; (c) voted centre position of ellipse; (d) histogram of distance $R_{e}$ pointing towards major and minor axes; (e) comparison of ellipse detected (green) with raw power Doppler image 


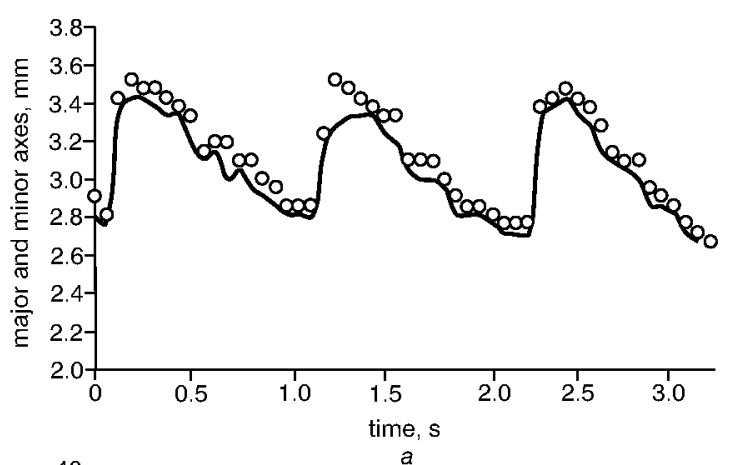

Table 1 Arterial diameter and strain predicted in healthy subjects

\begin{tabular}{lcccc}
\hline Artery & $\begin{array}{c}\text { Major } \\
\text { axis } a, \mathrm{~mm}\end{array}$ & $\begin{array}{c}\text { Minor } \\
\text { axis } b, \mathrm{~mm}\end{array}$ & $\begin{array}{c}\text { Diameter } \\
D, \mathrm{~mm}\end{array}$ & $\begin{array}{c}\text { Arterial strain } \\
\left(D_{s}-D_{d}\right) / D_{\text {mean }}, \%\end{array}$ \\
\hline CCA & $5.91(0.38)$ & $5.69(0.32)$ & $5.83(0.35)$ & $13.76(1.90)$ \\
BA & $4.29(0.28)$ & $3.77(0.48)$ & $4.03(0.36)$ & $16.74(4.56)$ \\
DPA & $2.86(0.12)$ & $2.51(0.32)$ & $2.68(0.22)$ & $15.52(5.44)$ \\
\hline
\end{tabular}

$D_{s}=$ peak systolic diameter; $D_{d}=$ end-diastolic diameter; $D_{\text {mean }}=$ mean diameter; CCA data are mean (standard variation) of nine male volunteers (mean age $24 \pm 6$ years). BA and DPA data were collected from five subjects (mean age 26 years)

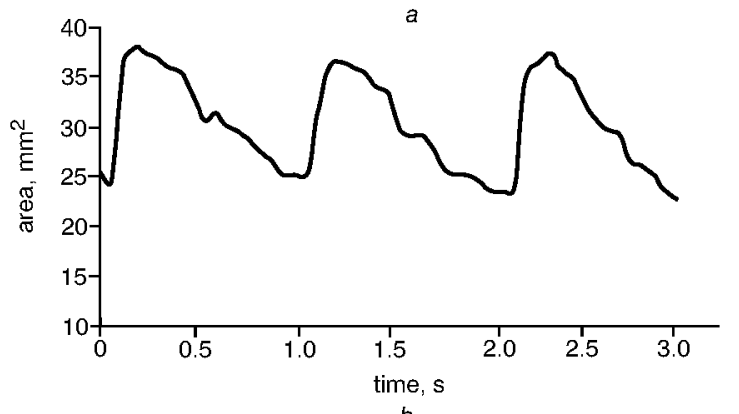

Fig. 4 CCA vessel distension: (a) change of $(\mathrm{O})$ major and (-) minor axes with cardiac cycle; (b) lumen area waveform
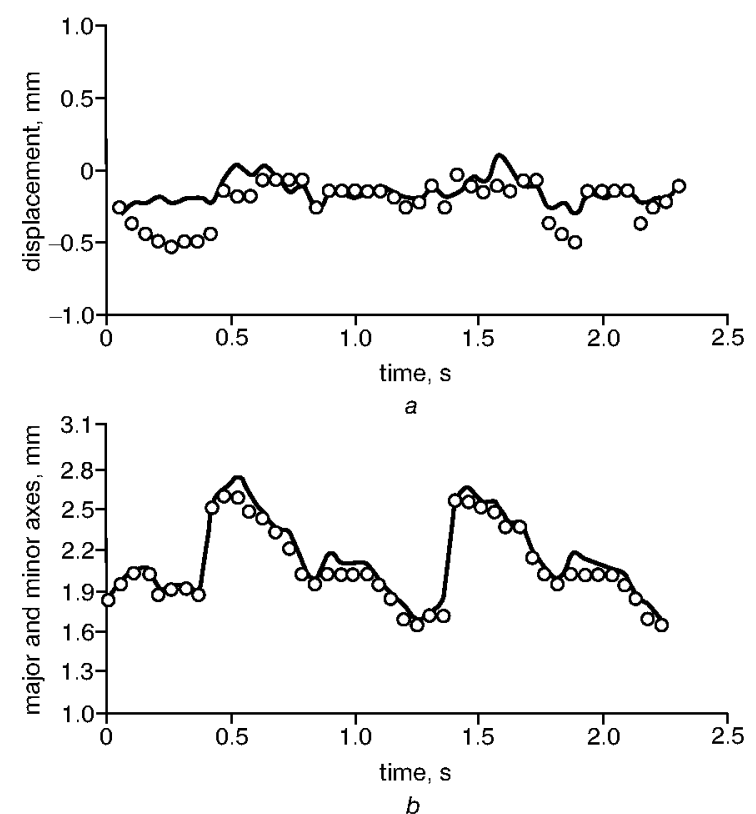

Fig. 5 BA vessel distension: (a) movement of ellipse centre: (O) $x$ direction; $(\longrightarrow)$-direction; $(b)$ change of $(\longrightarrow)$ major and (O) minor axes with cardiac cycle

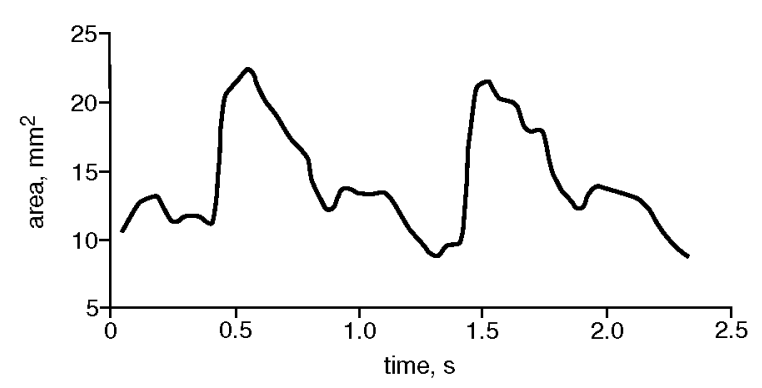

Fig. 6 Changes of $B A$ lumen area with cardiac cycle

from the interactions of the cardiac cycle and the surrounding muscular constraints. The vessel tended to move towards softer tissue. This provided a non-invasive tool to estimate the muscular contraction force acting on the arterial vessel locally and pointed towards possible external mechanisms for cardiovascular disease that are contributed by the surroundings.

In the current field of work, the power Doppler amplitude reflects the moving blood volume detected within the blood vessel better than colour Doppler imaging. However, the size of the blood vessel resulting from the image detection can depend on the power Doppler threshold selected. Therefore the ultrasound colour power angio (CPA) scale of the PDI should be sufficiently high ( $81-85 \%$, typically) to show the true arterial lumen sizes during various phases of the cardiac cycle. The body movement of the subject can cause severe noise in the PDI, and a probe fixture is needed to keep the ultrasound transducer securely positioned.

Based on our experience, the mean arterial vessel size detected with PDI is often under-estimated by $10-15 \%$. For peripheral arteries such as the BA and DPA, the PDI flow area in the diastolic phase often occupies less than the true lumen area. Therefore the mean vessel diameter can be slightly underestimated, and the vessel wall distension can be over-estimated. Caution should be shown when attempting to relate the changes in PDI area to the changes in vessel size of peripheral arteries (DEVERSON and EvANS, 2000). A lower PDI threshold value $(T h=6-10)$ is recommended for the BA and DPA. The measurement of the arterial distension waveform resulting from cardiac function using the lumen area is more sensitive than the vessel diameter, as the distension in all directions is included.

It should be noted that the time resolution is determined from the PDI frame rate and the persistence setting. In this study, for a vessel located at a depth of about $2 \mathrm{~cm}$, the PDI frame rate typically was only about $12-15$. Further, the vessel spatial resolution obtained using ultrasound image detection is not as good as that of the echo-tracking analogue signal. We can use high-density focusing of the ultrasound system to improve image resolution, and the PDI frame rate can also be made higher. With proper selection of ultrasound scanning mode and colourmap type, we can obtain a PDI frame rate as high as 30-40.

\section{Conclusions}

In the present study, a method that couples a gradient-based Hough transform and PDI is used to estimate an arterial vessel distension waveform of elliptic shape. Sequential frames of PDI were analysed using $\mathrm{C}++$ computer software that determined the centre of the ellipse, the major and minor axes and the arterial lumen area for each image frame. The arterial vessels were found to distend non-uniformly owing to the asymmetric action of the surrounding muscles and fatty tissues; this was not resolvable using traditional ultrasound techniques. 
The lumen area waveform of the peripheral artery is linearly related to the pulse pressure locally, for a young subject. We found that the continuous tracing of arterial lumen shape and position provides valuable information about the stiffness of the vessel itself and its surroundings. The present method may be a useful tool for the non-invasive assessment of the arterial waveform and the local mechanical properties of the arterial vessel.

Acknowledgments-This research was supported by grant NSC 892320-B-002-149 M08. The experiments were conducted in the Biomedical Ultrasound Research Laboratory of National Taiwan University Hospital; the use of facilities and technical assistance are gratefully acknowledged.

\section{References}

Aguado, A. S., Montiel, M. E., and Nixon, M. S. (1996): 'On using directional information for parameter space decomposition in ellipse detection', Patt. Recog., 29, pp. 369-381

Brands, P. J., Hoeks, A. P. G., Rutten, M. C. M., and Reneman, R. S. (1996): 'A noninvasive method to estimate arterial impedance by means of assessment of local diameter change and the local centerline blood flow velocity using ultrasound', Ultrasound Med. Biol., 22, pp. 895-905

DEVERSON, S., and EVANS, D. H. (2000): 'Using Doppler signal power to detect changes in vessel size: a feasibility study using a wall-less flow phantom', Ultrasound Med. Biol., 26, pp. 593-602

Griewing, B., Morgenstern, C., Driesner, F., Kallwellis, G., WALKER, M. L., and KESSLER, C. (1996): 'Cerebrovascular disease assessed by color-flow and power Doppler ultrasonography: comparison with digital subtraction angiography in internal carotid artery stenosis', Stroke, 27, pp. 95-100

Hoskins, P. R., Fish, P. J., MCDicken, W. N., and Moran, C. (1998): 'Developments in cardiovascular ultrasound. Part 2: arterial applications', Med. Biol. Eng. Comput., 36, pp. 259-269

Hough, P. V. C. (1962): 'Method and means for recognizing complex patterns'. US Patent 306954

ILLINGWORTH, J., and KitTLER, J. (1988): 'A survey of the Hough transform', Comput. Vis. Graph. Image Process., 44, pp. 87-116

JAIN, A. K. (1989): 'Fundamentals of digital image processing' (Prentice-Hall, 1989), pp. 348-357

LeI, Y., and WonG, K. C. (1999): 'Ellipse detection based on symmetry', Patt. Recogn. Lett., 20, pp. 41-47

Nagai, Y., Fleg, J. L., Kemper, M. K., Rywik, T. M., Earley, C. J., and METTER, E. J. R. (1999): 'Carotid arterial stiffiness as a surrogate for aortic stiffness: relationship between carotid artery pressure-strain elastic modulus and aortic pulse wave velocity', Ultrasound Med. Biol., 25, pp. 181-188

NAIR, P. S., and SAUNDERS, A. T., Jr. (1996): 'Hough transform based ellipse detection algorithm', Patt. Recog. Lett., 17, pp. 777-784
ShaU, Y. W., Wang, C. L., ShIEH, J. Y., and HsU, T. C. (1999): 'Noninvasive assessment of the viscoelasticity of peripheral arteries', Ultrasound Med. Biol., 25, pp. 1377-1388

StadLer, R. W., KARL, W. C., and LeES, R. S. (1996): 'New methods for arterial diameter measurement from B-mode images', Ultrasound Med. Biol., 22, pp. 25-34

STADLER, R. W., TAYloR, J. A., and LeES, R. S. (1997): 'Comparison of B-mode, M-mode and echo-tracking methods for measurement of the arterial distension waveform', Ultrasound Med. Biol., 23, pp. $879-887$

Steinke, W., MEAirs, S., RIES, S., and HENNERICI, M. (1996): 'Sonographic assessment of carotid artery stenosis: Comparison of power Doppler imaging and color Doppler flow imaging', Stroke, 27, pp. 91-94

Steinke, W., Ries, S., Artemis, N., Schwartz, A., and Hennerici, M. (1997): 'Power Doppler imaging of carotid artery stenosis: comparison with color Doppler flow imaging and angiography', Stroke, 28, pp. 1981-1987

WOLF, K. J., and FOBBE, F. (1994): 'Color duplex sonography: principles and clinical applications' (Thieme Medical Publishers Inc., New York)

YIP, R. K., TAM, P. K., and LEUNG, D. N. (1992): 'Modification of Hough transform for circles and ellipses detection using a 2dimensional array', Patt. Recog., 25, pp. 1007-1022

\section{Authors' biographies}

SHU-MEI WU received her BS in Electronic Engineering in 1990 and MS in Electrical Engineering in 1994 from National Taiwan University of Science and Technology. She is currently a $\mathrm{PhD}$ candidate in the Electrical Engineering Department of National Taiwan University and a lecturer at the Jin-Wen Institute of Technology. Her research focuses on medical image processing and bio-signal processing.

Yio-Wha ShaU received his MS and $\mathrm{PhD}$ in Aerospace Engineering from the University of Texas at Austin, in 1986 and 1990, respectively. He is an associate professor in the Institute of Applied Mechanics of National Taiwan University. His research interests include the medical imaging processing, design of artificial organs, and the development of non-invasive diagnostic tools for clinical sciences.

FoK-CHING CHONG obtained his BS from the Electrical Engineering Department of National Taiwan University in 1971. He is currently an associate professor in the same department. He is interested in biomedical signal processing, simulation, system analysis and design.

Fon-Jou HsieH, MD, $\mathrm{PhD}$, is a professor in the Department of Obstetrics and Gynecology of National Taiwan University Medical College. He is also the associate director of the Institute of Clinical Medicine. His research interests include medical ultrasound and genetics. 